\title{
Performancemessung im Eigenhandel mittels Value at Risk - Teil 2: Lösungen
}

\author{
Pascal Thomas, Friedrich Then Bergh und Raimund Schirmeister
}

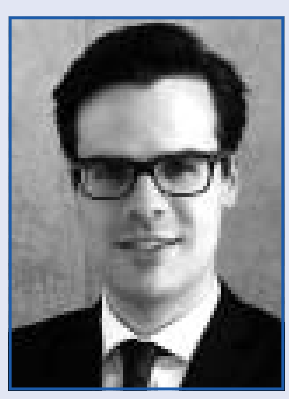

Pascal Thomas, M.Sc. ist Wissenschaftlicher Mitarbeiter am Lehrstuhl für Betriebswirtschaftslehre, insbes. Finanzierung und Investition der HeinrichHeine-Universität Düsseldorf. Bevorzugte Forschungsgebiete: Risikomanagement und Compliance.

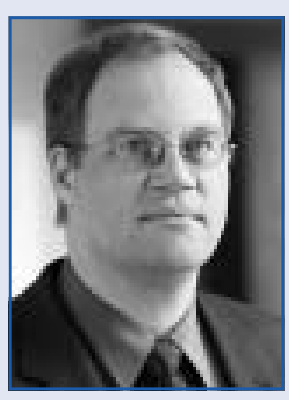

Prof. Dr. Friedrich Then Bergh ist Leiter des Studiengangs BWL - Finanzdienstleistungen an der DHBW Ravensburg. Bevorzugte Forschungsgebiete: Corporate Finance, Accounting und Riskmanagement.

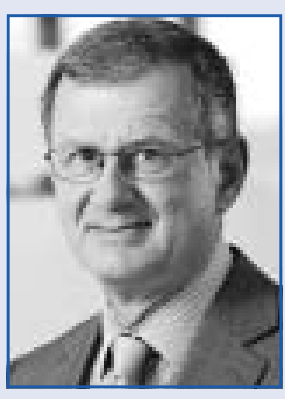

Prof. Dr. Raimund Schirmeister ist Inhaber des Lehrstuhls für Betriebswirtschaftslehre, insbes. Finanzierung und Investition der Heinrich-Heine-Universität Düsseldorf. Bevorzugte Forschungsgebiete: Investition, Unternehmensrechnung und Finanzmanagement.

Der Value at Risk (VaR) ermöglicht eine Effizienzsteigerung sowohl der Risikomessung als auch der Risikosteuerung und -kontrolle. Innerhalb des Eigenhandels einer Bank, als Ausgangssituation dargestellt im letzten WiSt-Heft, Nr. 6/2016, S. 328 f., wird unter der Betrachtung konzeptionell divergierender Berechnungsmethoden die Verwendbarkeit des Value at Risk für einzelne Aktienkursrisiken sowie für Aktienportfolios aufgezeigt. Zugleich werden Möglichkeiten der risikoadjustierten Renditemessung auf Basis des Value at Risk diskutiert.

Stichwörter: Risikomanagement, Value at Risk, Risikoadjustierte Renditemaße, Portfoliotheorie

\section{Aufgabe 1}

a) Modellanalytischer Ansatz:

Zunächst ergeben sich für die vorliegenden Aktien die in Tab. 1 und Tab. 2 enthaltenen Renditen.

Unter Verwendung von Formel (1) lassen sich folgende Standardabweichungen und VaR-Werte schätzen:

- BelgaCola (BC):

$\hat{\sigma}^{B C}=\sqrt{\frac{1}{19} \times 0,034265-\frac{1}{380} \times 0,039221^{2}}=0,04241879$

Da $\Phi(0,99)=2,326348$, folgt für den Ein-Tages-VaR (vgl. Formel (1) des oben genannten Beitrags im letzten WiStHeft):

$$
\begin{aligned}
\operatorname{VaR}_{0,99}^{\mathrm{BC}} & =2,326348 \times 0,04241879 \times 500.000 \\
& =49.340,43364
\end{aligned}
$$

- Énergie Belgique (EB):

$$
\begin{aligned}
\hat{\sigma}^{E B} & =\sqrt{\frac{1}{19} \times 0,000064-\frac{1}{380} \times(-0,002002)^{2}} \\
& =0,001825919
\end{aligned}
$$

sowie

$$
\begin{aligned}
\mathrm{VaR}_{0,99}^{\mathrm{EB}} & =2,326348 \times 0,001825919 \times 500.000 \\
& =2.123,861507
\end{aligned}
$$

\section{b) Historische Simulation:}

Auf Grundlage der historischen Kursentwicklung ergeben sich die in Tab. 3 und Tab. 4 dargestellten möglichen Wertänderungen (Szenario $\mathrm{t} \hat{=}$ Wertänderung des Handelstags $\mathrm{t}$ ) für den nächsten Handelstag.

Aus der geordneten Rangfolge der simulierten Wertänderungen der jeweiligen Position (vgl. Tab. 5 und Tab. 6) kann mithilfe linearer Interpolation der allgemeinen Form

$f_{0} \frac{x_{1}-x}{x_{1}-x_{0}}+f_{1} \frac{x-x_{0}}{x_{1}-x_{0}}$

dann das $1 \%$-Perzentil, folglich also der VaR, ermittelt werden.

\section{- BelgaCola:}

$\Rightarrow \operatorname{VaR}_{0,99}^{\mathrm{BC}}=-32.027,3632 \frac{0,05263158-0,01}{0,05263158-0}$ 
Tab. 1: Renditeberechnung der BelgaCola-Aktien

\begin{tabular}{|c|c|c|c|c|}
\hline Handelstag & $\begin{array}{c}\text { Schlusskurs } \\
S_{\mathrm{t}}(\text { in } € \text { ) }\end{array}$ & $\begin{array}{c}\text { Kursverhältnis } \\
\mathbf{S}_{\mathrm{t}} / \mathbf{S}_{\mathrm{t}-1}\end{array}$ & $\begin{array}{c}\mathbf{u}_{t} \\
=\ln \left(S_{t} / S_{t-1}\right)\end{array}$ & $u_{t}^{2}$ \\
\hline 0 & 17,00 & - & - & - \\
\hline 1 & 17,15 & $\begin{array}{l}17,15 / 17,00 \\
=1,0088235 \\
\end{array}$ & 0,0087848 & 0,0000772 \\
\hline 2 & 16,59 & 0,9673469 & $-0,0331981$ & 0,0011021 \\
\hline 3 & 16,08 & 0,9692586 & $-0,0312238$ & 0,0009749 \\
\hline 4 & 15,05 & 0,9359453 & $-0,0661983$ & 0,0043822 \\
\hline 5 & 17,01 & 1,1302326 & 0,1224234 & 0,0149875 \\
\hline 6 & 17,52 & 1,0299824 & 0,0295417 & 0,0008727 \\
\hline 7 & 17,69 & 1,0097032 & 0,0096564 & 0,0000932 \\
\hline 8 & 18,34 & 1,0367439 & 0,0360850 & 0,0013021 \\
\hline 9 & 17,87 & 0,9743730 & $-0,0259611$ & 0,0006740 \\
\hline 10 & 18,68 & 1,0453274 & 0,0443301 & 0,0019652 \\
\hline 11 & 19,03 & 1,0187366 & 0,0185632 & 0,0003446 \\
\hline 12 & 19,00 & 0,9984235 & $-0,0015777$ & 0,0000025 \\
\hline 13 & 17,98 & 0,9463158 & $-0,0551790$ & 0,0030447 \\
\hline 14 & 17,44 & 0,9699666 & $-0,0304936$ & 0,0009299 \\
\hline 15 & 17,21 & 0,9868119 & $-0,0132758$ & 0,0001762 \\
\hline 16 & 17,56 & 1,0203370 & 0,0201330 & 0,0004053 \\
\hline 17 & 16,94 & 0,9646925 & $-0,0359459$ & 0,0012921 \\
\hline 18 & 16,74 & 0,9881936 & $-0,0118766$ & 0,0001411 \\
\hline 19 & 17,23 & 1,0292712 & 0,0288510 & 0,0008324 \\
\hline 20 & 17,68 & 1,0261172 & 0,0257820 & 0,0006647 \\
\hline$\Sigma$ & & & 0,0392207 & 0,0342646 \\
\hline
\end{tabular}

$$
\begin{aligned}
& +(-26.842,1053) \frac{0,01-0}{0,05263158-0} \\
& =-31.042,1642
\end{aligned}
$$

\section{- Énergie Belgique:}

$$
\begin{aligned}
\Rightarrow \mathrm{VaR}_{0,99}^{\mathrm{EB}} & =-2.381,3182 \frac{0,05263158-0,01}{0,05263158-0} \\
& +(-1.380,1564) \frac{0,01-0}{0,05263158-0} \\
& =-2.191,0974
\end{aligned}
$$

Die historische Simulation leidet hier unter den extrem kurzen Kurshistorien. Während der VaR der Énergie Belgique-Aktie unter beiden Berechnungsmethoden betragsmäßig ähnliche Werte annimmt, erbringt die historische Simulation in Bezug auf die BelgaCola-Position einen weit geringeren VaR. Ursächlich hierfür ist die sehr hohe Standardabweichung der Aktie, die mehrheitlich aus positiven Wertänderungen, insbesondere der exorbitant hohen Wertänderung auf Rang eins, resultiert. Der mit dem Varianz-Kovarianz-Ansatz ermittelte VaR berücksichtigt dies infolge seiner Berechnungsweise: In die Standardabweichung gehen sämtliche Wertänderungen gleich gewichtet ein. Demgegenüber sind bei der historischen Simulation aufgrund des hier limitierend kurzen Beobachtungszeitraums ähnlich hohe negative Abweichungen schlicht nicht enthalten. Die Limitation der Betrachtung auf die schlechtesten Wertänderungen ist mithin als entscheidendes Problem der historischen Simulation anzusehen, das grundsätzlich auch bei längerer Kurshistorie erhalten bleibt.

\section{Aufgabe 2}

Der Korrelationskoeffizient der beiden vorliegend betrachteten Aktien beträgt $\rho=0,04405968$, sodass sich die Standardabweichung des Portfolios ergibt als:

$$
\begin{aligned}
\sigma_{P} & =\sqrt{\begin{array}{c}
0,5^{2} \times 0,042419^{2}+0,5^{2} \times 0,001826^{2}+ \\
2 \times 0,5 \times 0,5 \times 0,042419 \times 0,001826 \times 0,044060
\end{array}} \\
& =0,021269185
\end{aligned}
$$

Daraus lässt sich folgender VaR des Portfolios ableiten:

$$
\begin{aligned}
\operatorname{VaR}_{0,99}^{\mathrm{P}} & =2,326348 \times 0,021269185 \times 1.000 .000 \\
& =49.479,52599
\end{aligned}
$$




\begin{tabular}{|c|c|c|c|c|}
\hline Handelstag & $\begin{array}{c}\text { Schlusskurs } \\
\mathbf{S}_{\mathbf{t}}(\mathbf{i n} \boldsymbol{\epsilon})\end{array}$ & $\begin{array}{c}\text { Kursverhältnis } \\
\mathbf{S}_{\mathbf{t}} / \mathbf{S}_{\mathbf{t}-1}\end{array}$ & $\begin{array}{c}\mathbf{u}_{\mathbf{t}} \\
\mathbf{l n}\left(\mathbf{S}_{\mathbf{t}} / \mathbf{S}_{\mathbf{t}-1}\right)\end{array}$ & \multicolumn{1}{c|}{$\mathbf{u}_{\mathbf{t}}{ }^{2}$} \\
\hline 0 & 65,00 & - & - & - \\
\hline 1 & 64,89 & 0,9983077 & $-0,0016937$ & 0,0000029 \\
\hline 2 & 64,92 & 1,0004623 & 0,0004622 & 0,0000002 \\
\hline 3 & 64,97 & 1,0007702 & 0,0007699 & 0,0000006 \\
\hline 4 & 65,01 & 1,0006157 & 0,0006155 & 0,0000004 \\
\hline 5 & 65,13 & 1,0018459 & 0,0018442 & 0,0000034 \\
\hline 6 & 65,21 & 1,0012283 & 0,0012276 & 0,0000015 \\
\hline 7 & 65,03 & 0,9972397 & $-0,0027641$ & 0,0000076 \\
\hline 8 & 65,09 & 1,0009227 & 0,0009222 & 0,0000009 \\
\hline 9 & 64,78 & 0,9952374 & $-0,0047740$ & 0,0000228 \\
\hline 10 & 64,77 & 0,9998456 & $-0,0001544$ & 0,0000000 \\
\hline 11 & 64,74 & 0,9995368 & $-0,0004633$ & 0,0000002 \\
\hline 12 & 64,83 & 1,0013902 & 0,0013892 & 0,0000019 \\
\hline 13 & 65,09 & 1,0040105 & 0,0040025 & 0,0000160 \\
\hline 14 & 65,12 & 1,0004609 & 0,0004608 & 0,0000002 \\
\hline 15 & 65,08 & 0,9993857 & $-0,0006144$ & 0,0000004 \\
\hline 16 & 64,99 & 0,9986171 & $-0,0013839$ & 0,0000019 \\
\hline 17 & 64,91 & 0,9987690 & $-0,0012317$ & 0,0000015 \\
\hline 18 & 64,90 & 0,9998459 & $-0,0001541$ & 0,0000000 \\
\hline 19 & 64,93 & 1,0004622 & 0,0004621 & 0,0000002 \\
\hline 20 & 64,87 & 0,9990759 & $-0,0009245$ & 0,0000009 \\
\hline$\sum$ & & & $-0,0020020$ & 0,0000635 \\
\hline
\end{tabular}

Tab. 2: Renditeberechnung der Énergie Belgique-Aktien
Gegenüber der Summe der VaR-Werte der beiden Aktien, 49.340,43364 $€+2.123,861507 €=51.464,29515 €$, konnte durch die Portfoliobildung offensichtlich ein Risikodiversifikationseffekt in Höhe von 51.464,29515 €49.479,52599 $€=1.984,769157 €$ erreicht werden. Dies entspricht einer Reduktion des Gesamt-VaR um 3,86 \%.

Welchen Anteil jede der beiden betrachteten Aktien an diesem Diversifikationseffekt und damit am Portfolio-VaR hat, lässt sich mittels des CoVaR ermitteln.

Mithilfe von Tab. 2 der Aufgabenstellung (vgl. letztes WiSt-Heft, Nr. 6/2016, S. 328 f.) und der Formel

$\mathrm{CoVaR}_{\mathrm{i}}=\operatorname{VaR}_{\mathrm{P}} \times \rho_{i P}$

ergeben sich folgende Werte:

$\mathrm{CoVaR}_{\mathrm{BC}}=49.340,43 \times 0,99908=49.295,04$

$\mathrm{CoVaR}_{\mathrm{EB}}=2.123,86 \times 0,08686=184,53$

Aus der Summe beider CoVaR-Werte ergibt sich dann, rundungsbedingt geringfügig abweichend, der bereits ermittelte VaR des Portfolios in Höhe von 49.479,58 €.

Während innerhalb des Portfolios der VaR der BelgaColaAktie kaum durch den Diversifikationseffekt reduziert werden kann, entfällt dieser Effekt nahezu vollständig auf die Aktie der Énergie Belgique, deren VaR durch die Port- foliobildung mithin erheblich gesenkt werden konnte. Demnach ist fast ausschließlich der VaR der BelgaColaAktie ausschlaggebend für den Portfolio-VaR.

\section{Aufgabe 3}

Da im vorliegenden Fall der RoRaC im Portfoliozusammenhang ermittelt werden soll, bietet es sich an, anstelle des VaR den CoVaR als nach der Diversifikation verbleibendes Risikokapital heranzuziehen, sodass gilt (vgl. Formel (18) des Beitrags):

$\mathrm{IstRoRaC}_{i}=\frac{\left(\bar{\mu}_{i}-r\right) \times I+\mathrm{D}_{i}}{\mathrm{CoVaR}_{i}}$

mit $\mu_{i}=$ durchschnittliche Rendite der Aktie i,

$\mathrm{D}_{i}=$ Dividendenzahlung der Aktie $\mathrm{i}$,

$\mathrm{r}=$ risikoloser Zins und

$I=$ Investitionsvolumen.

Aus den Tab. 1 und 2 lässt sich jeweils die durchschnittliche Tagesrendite der beiden Aktien berechnen. Es ergibt sich:

$\bar{\mu}_{B C}^{T a g}=0,001961035$

$\bar{\mu}_{E B}^{T a g}=-0,0001001$ 


\begin{tabular}{|c|c|c|c|c|c|}
\hline Szenario & $\begin{array}{l}\text { Ausgangswert } \\
\text { (in } €)\end{array}$ & $\begin{array}{c}\text { (1) } \\
\text { Relative } \\
\text { Wertänderung }\end{array}$ & $\begin{array}{l}\text { Simulierter } \\
\text { Wert (in } € \text { ) }\end{array}$ & $\begin{array}{c}\text { (2) } \\
\text { Simulierte Rendite } \\
\text { (1) }-1 \\
\end{array}$ & $\begin{array}{c}\text { (2) x } 500.000 € \\
\text { Simulierte Wertänderung der Position } \\
\text { (in } € \text { ) }\end{array}$ \\
\hline 0 & 17,68 & - & - & - & - \\
\hline 1 & 17,68 & 1,0088235 & 17,8360000 & 0,0088235 & $0,00882353 \times 500.000=4.411,76471$ \\
\hline 2 & 17,68 & 0,9673469 & 17,1026939 & $-0,0326531$ & $-16.326,5306$ \\
\hline 3 & 17,68 & 0,9692586 & 17,1364919 & $-0,0307414$ & $-15.370,7052$ \\
\hline 4 & 17,68 & 0,9359453 & 16,5475124 & $-0,0640547$ & $-32.027,3632$ \\
\hline 5 & 17,68 & 1,1302326 & 19,9825116 & 0,1302326 & $65.116,2791$ \\
\hline 6 & 17,68 & 1,0299824 & 18,2100882 & 0,0299824 & $14.991,1817$ \\
\hline 7 & 17,68 & 1,0097032 & 17,8515525 & 0,0097032 & $4.851,59817$ \\
\hline 8 & 17,68 & 1,0367439 & 18,3296326 & 0,0367439 & $18.371,9616$ \\
\hline 9 & 17,68 & 0,9743730 & 17,2269138 & $-0,0256270$ & $-12.813,5224$ \\
\hline 10 & 17,68 & 1,0453274 & 18,4813878 & 0,0453274 & $22.663,6821$ \\
\hline 11 & 17,68 & 1,0187366 & 18,0112634 & 0,0187366 & $9.368,30835$ \\
\hline 12 & 17,68 & 0,9984235 & 17,6521282 & $-0,0015765$ & $-788,229112$ \\
\hline 13 & 17,68 & 0,9463158 & 16,7308632 & $-0,0536842$ & $-2.6842,1053$ \\
\hline 14 & 17,68 & 0,9699666 & 17,1490100 & $-0,0300334$ & $-15.016,6852$ \\
\hline 15 & 17,68 & 0,9868119 & 17,4468349 & $-0,0131881$ & $-6.594,03670$ \\
\hline 16 & 17,68 & 1,0203370 & 18,0395584 & 0,0203370 & $10.168,5067$ \\
\hline 17 & 17,68 & 0,9646925 & 17,0557631 & $-0,0353075$ & $-17.653,7585$ \\
\hline 18 & 17,68 & 0,9881936 & 17,4712633 & $-0,0118064$ & $-5.903,18772$ \\
\hline 19 & 17,68 & 1,0292712 & 18,1975149 & 0,0292712 & $14.635,6033$ \\
\hline 20 & 17,68 & 1,0261172 & 18,1417528 & 0,0261172 & $13.058,6187$ \\
\hline
\end{tabular}

Tab. 3: Historische Simulation für die BelgaCola-Aktie

Bei 250 Handelstagen lassen sich damit folgende jährliche Durchschnittsrenditen prognostizieren:

$\bar{\mu}_{B C}=0,001961 \times 250=0,49026$

$\bar{\mu}_{E B}=-0,0001 \times 250=-0,02503$

Abzüglich des risikolosen Zinses in Höhe von $\mathrm{r}=0,0025$ ergibt sich damit folgender Nettoerfolg für BelgaCola NV, bezogen auf das Investitionsvolumen von $500.000 €$ :

Nettoerfolg ${ }_{B C}=0,48776 \times 500.000=243.880,00$.

Bei der Énergie Belgique S.A. erfolgt zusätzlich eine prognostizierte Ausschüttung in Höhe von 1,82 €/Aktie, sodass sich bei einem Investitionsvolumen von $500.000 € \mathrm{zu}$ einem Kurs von $64,87 €$ eine gehaltene Anzahl an Aktien von 7.707,72314 ergibt. Insgesamt folgt daraus ein Ausschüttungsgewinn der gehaltenen Position in Höhe von 14.028,06 $€$. Hieraus wiederum resultiert folgender Nettoerfolg:

$$
\begin{aligned}
\text { Nettoerfolg }_{\text {EB }} & =-0,02753 \times 500.000+14.028,06 \\
& =263,06
\end{aligned}
$$

Die beiden oben angegebenen Werte für die CoVaRs auf ein Jahr bezogen lauten:

$$
\begin{aligned}
& \mathrm{CoVaR}_{\mathrm{BC}}^{\mathrm{Jahr}}=49.295,04 \times \sqrt{250}=779.423,02 \\
& \mathrm{CoVaR}_{\mathrm{EB}}^{\mathrm{Jahr}}=184,53 \times \sqrt{250}=2.917,74
\end{aligned}
$$

Da jedoch lediglich ein Betrag von $500.000 €$ in die Aktien der BelgaCola NV investiert wurde, wird der CoVaR entsprechend auf

$\mathrm{CoVaR}_{\mathrm{BC}}^{\mathrm{Jahr}}=500.000$

beschränkt, da kein über das eingesetzte Kapital hinausgehender Verlust möglich ist.

Mittels dieser Werte können nun die RoRaC der beiden Aktien wie folgt berechnet werden:

IstRoRaC $_{B C}=\frac{243.880,00}{500.000}=0,48776$
IstRoRaC $_{\mathrm{EB}}=\frac{263,0561}{2.917,735753}=0,09016$

Offensichtlich entspricht der RoRaC der BelgaCola $\mathrm{NV}$ aufgrund der fehlenden Dividendenzahlung sowie der Verwendung der gesamten Investitionssumme als Risikokapital gerade der oben ermittelten Überschussrendite der Aktie. Demgegenüber ändert sich die Einschätzung der Énergie Belgique-Aktien deutlich, wenn man deren geringe Risikobehaftung berücksichtigt: Aufgrund ihres geringen Risiko kann die Aktie risikoadjustiert eine durchaus ordentliche Rendite erzielen. 


\begin{tabular}{|c|c|c|c|c|c|}
\hline Szenario & $\begin{array}{l}\text { Ausgangswert } \\
\text { (in } €)\end{array}$ & $\begin{array}{c}\text { (1) } \\
\text { Relative } \\
\text { Wertänderung }\end{array}$ & $\begin{array}{l}\text { Simulierter } \\
\text { Wert (in } € \text { ) }\end{array}$ & $\begin{array}{l}\text { (2) } \\
\text { Simulierte Rendite } \\
\text { (1) }-1\end{array}$ & $\begin{array}{c}\text { (2) } \times 500.000 € \\
\text { Simulierte Wertänderung der } \\
\text { Position } \\
\text { (in } €)\end{array}$ \\
\hline 0 & 64,87 & - & - & - & - \\
\hline 1 & 64,87 & 0,9983077 & 64,7602200 & $-0,0016923$ & $-846,153846$ \\
\hline 2 & 64,87 & 1,0004623 & 64,8999908 & 0,0004623 & 231,160425 \\
\hline 3 & 64,87 & 1,0007702 & 64,9199615 & 0,0007702 & 385,089341 \\
\hline 4 & 64,87 & 1,0006157 & 64,9099384 & 0,0006157 & 307,834385 \\
\hline 5 & 64,87 & 1,0018459 & 64,9897416 & 0,0018459 & 922,934933 \\
\hline 6 & 64,87 & 1,0012283 & 64,9496806 & 0,0012283 & 614,156303 \\
\hline 7 & 64,87 & 0,9972397 & 64,6909385 & $-0,0027603$ & $-1.380,15642$ \\
\hline 8 & 64,87 & 1,0009227 & 64,9298524 & 0,0009227 & 461,325542 \\
\hline 9 & 64,87 & 0,9952374 & 64,5610478 & $-0,0047626$ & $-2.381,31817$ \\
\hline 10 & 64,87 & 0,9998456 & 64,8599861 & $-0,0001544$ & $-77,1843161$ \\
\hline 11 & 64,87 & 0,9995368 & 64,8399537 & $-0,0004632$ & $-231,588698$ \\
\hline 12 & 64,87 & 1,0013902 & 64,9601807 & 0,0013902 & 695,088044 \\
\hline 13 & 64,87 & 1,0040105 & 65,1301604 & 0,0040105 & $2.005,24449$ \\
\hline 14 & 64,87 & 1,0004609 & 64,8998986 & 0,0004609 & 230,450146 \\
\hline 15 & 64,87 & 0,9993857 & 64,8301536 & $-0,0006143$ & $-307,125307$ \\
\hline 16 & 64,87 & 0,9986171 & 64,7802904 & $-0,0013829$ & $-691,456669$ \\
\hline 17 & 64,87 & 0,9987690 & 64,7901477 & $-0,0012310$ & $-615,479305$ \\
\hline 18 & 64,87 & 0,9998459 & 64,8600062 & $-0,0001541$ & $-77,0297335$ \\
\hline 19 & 64,87 & 1,0004622 & 64,8999861 & 0,0004622 & 231,124807 \\
\hline 20 & 64,87 & 0,9990759 & 64,8100554 & $-0,0009241$ & $-462,036039$ \\
\hline
\end{tabular}

Tab. 4: Historische Simulation für die Énergie Belgique-Aktie

Gemäß der Gewichtung der beiden Aktien im Portfolio anhand des CoVaR,

$x_{B C}=\frac{500.000}{502 \cdot 917,74}=0,9942$

$x_{E B}=\frac{2 \cdot 917,74}{502 \cdot 917,74}=0,00580$,

ergibt sich der RoRaC des Portfolios als

IstRoRaC $_{\mathrm{P}}=0,9942 \times 0,48776+0,00580 \times 0,09016=$ 0,48545 .

Gemessen am Risikogehalt wird das Portfolio von der BelgaCola-Aktie beherrscht, die zweifelsfrei vorteilhaft erscheinende Aktie geht indes mit exorbitanten Risiken einher. Die weit stabiler verlaufende Énergie Belgique-Aktie erscheint risikoadjustiert ebenfalls vorteilhaft. Der durch die Standardabweichung sowie den VaR abgebildete positive Diversifikationseffekt lässt sich auf Grundlage des RoRaC nicht widerspiegeln, vielmehr würde hier die Vorteilhaftigkeit einer vollständigen Investition in die risikoreiche BelgaCola-Aktie suggeriert werden. Die Portfoliozusammensetzung muss daher in Abhängigkeit der Risikopräferenz bzw. -tragfähigkeit erfolgen.
Betrachtet man zusätzlich den (Ist)RaRoC der Investitionen in die BelgaCola und in die Énergie Belgique, so stellt sich die Situation beider Investments - sofern man einen ZielRoRaC von $20 \%$ unterstellt - gemäß Tab. 7 dar.

Für das Portfolio folgt damit ein RaRoC von

$$
\begin{aligned}
\operatorname{RaRoC}_{\mathrm{P}} & =\left(\text { IstRoRaC }_{\mathrm{P}}\right)-\left(\text { ZielRoRaC }_{\mathrm{P}}\right) \\
& =0,48545-0,2 \\
& =x_{B C} \times \operatorname{RaRoC}_{\mathrm{BC}}+x_{E B} \times \operatorname{RaRoC}_{\mathrm{EB}} \\
& =0,9942 \times 0,28776+0,00580 \times(-0,10984) \\
& =0,28545
\end{aligned}
$$

Es wird offensichtlich, dass sich für das Investment in die Aktie der Énergie Belgique die Beurteilung der Vorteilhaftigkeit je nach Perspektive ändert:

Ergibt sich für die prognostizierte jährliche Durchschnittsrendite (ausschließlich auf Basis der Kursentwicklung) ein negativer Wert von

$\bar{\mu}_{E B}=-0,0001 \times 250=-0,02503<0$,

so errechnet sich unter Berücksichtigung der Dividendenzahlungen ein positiver IstRoRaC

IstRoRaC $_{\mathrm{EB}}=\frac{263,06}{2.917,74}=0,09016>0$, 


\begin{tabular}{|c|c|c|}
\hline $\begin{array}{c}\text { Simulierte } \\
\text { Wertänderung der } \\
\text { Position (in } € \text { ) } \\
\end{array}$ & Rang & $\begin{array}{c}\text { Prozent } \\
(1 / 19 \\
=5,2632 \%) \\
\end{array}$ \\
\hline $65.116,2791$ & 1 & $100,0000 \%$ \\
\hline $22.663,6821$ & 2 & $94,7376 \%$ \\
\hline $18.371,9616$ & 3 & $89,4744 \%$ \\
\hline $14.991,1817$ & 4 & $84,2112 \%$ \\
\hline $14.635,6033$ & 5 & $78,9480 \%$ \\
\hline $13.058,6187$ & 6 & $73,6848 \%$ \\
\hline $10.168,5067$ & 7 & $68,4216 \%$ \\
\hline $9.368,3084$ & 8 & $63,1584 \%$ \\
\hline $4.851,5982$ & 9 & $57,8952 \%$ \\
\hline $4.411,7647$ & 10 & $52,6320 \%$ \\
\hline$-788,2291$ & 11 & $47,3688 \%$ \\
\hline$-5.903,1877$ & 12 & $42,1056 \%$ \\
\hline$-6.594,0367$ & 13 & $36,8424 \%$ \\
\hline$-12.813,5224$ & 14 & $31,5792 \%$ \\
\hline$-15.016,6852$ & 15 & $26,3160 \%$ \\
\hline$-15.370,7052$ & 16 & $21,0528 \%$ \\
\hline$-16.326,5306$ & 17 & $15,7896 \%$ \\
\hline$-17.653,7585$ & 18 & $10,5264 \%$ \\
\hline$-26.842,1053$ & 19 & $5,2632 \%$ \\
\hline$-32.027,3632$ & 20 & $0,0000 \%$ \\
\hline
\end{tabular}

Tab. 5: Perzentile der simulierten Wertänderungen der BelgaColaAktie

\begin{tabular}{|c|c|c|}
\hline $\begin{array}{c}\text { Simulierte } \\
\text { Wertänderung der } \\
\text { Position (in } € \text { ) } \\
\end{array}$ & Rang & $\begin{aligned} & \text { Prozent } \\
&(1 / 19 \\
&=5,2632 \%) \\
&\end{aligned}$ \\
\hline $2.005,2445$ & 1 & $100,0000 \%$ \\
\hline 922,9349 & 2 & $94,7376 \%$ \\
\hline 695,0880 & 3 & $89,4744 \%$ \\
\hline 614,1563 & 4 & $84,2112 \%$ \\
\hline 461,3255 & 5 & $78,9480 \%$ \\
\hline 385,0893 & 6 & $73,6848 \%$ \\
\hline 307,8344 & 7 & $68,4216 \%$ \\
\hline 231,1604 & 8 & $63,1584 \%$ \\
\hline 231,1248 & 9 & $57,8952 \%$ \\
\hline 230,4501 & 10 & $52,6320 \%$ \\
\hline$-77,0297$ & 11 & $47,3688 \%$ \\
\hline$-77,1843$ & 12 & $42,1056 \%$ \\
\hline$-231,5887$ & 13 & $36,8424 \%$ \\
\hline$-307,1253$ & 14 & $31,5792 \%$ \\
\hline$-462,0360$ & 15 & $26,3160 \%$ \\
\hline$-615,4793$ & 16 & $21,0528 \%$ \\
\hline$-691,4567$ & 17 & $15,7896 \%$ \\
\hline$-846,1538$ & 18 & $10,5264 \%$ \\
\hline$-1.380,1564$ & 19 & $5,2632 \%$ \\
\hline$-2.381,3182$ & 20 & $0,0000 \%$ \\
\hline
\end{tabular}

Tab. 6: Perzentile der simulierten Wertänderungen der Énergie Belgique-Aktie

\begin{tabular}{|c|c|c|c|}
\hline & & $B C$ & $E B$ \\
\hline & (a) Volumen & $500.000,00$ & $500.000,00$ \\
\hline & (b) CoVaR ${ }^{\mathrm{Jahr}}$ & $500.000,00$ & $2.917,74$ \\
\hline & (c) Nettoertrag & $243.880,00$ & 263,06 \\
\hline & (d) IstRoRaC [(c)/(b)] & 0,48776 & 0,09016 \\
\hline & (e) ZielRoRaC & 0,20 & 0,20 \\
\hline & (f) Ziel-Ergebnis [(e)x(b)] & $100.000,00$ & 583,55 \\
\hline & (g) Risk adjusted Return [(c)-(f)] & $143.880,00$ & $-320,49$ \\
\hline Tab. 7: Risikoadjustierte Rendi- & (h) $\operatorname{RaRoC}[(\mathrm{g}) /(\mathrm{b})$ bzw. (d)-(e)] & 0,28776 & $-0,10984$ \\
\hline
\end{tabular}

aber bei einem angenommenen ZielRoRaC von 0,2 wiederum ein negativer RaRoC:

$\mathrm{RaRoC}_{\mathrm{EB}}=\frac{263,06-(0,2 \times 2.917,74)}{2.917,74}=-0,10984<0$.
Literatur

Hartmann-Wendels, T., A. Pfingsten, M. Weber, Bankbetriebslehre, 6. Aufl., Berlin, Heidelberg 2015.

Hull, J., Risikomanagement, 2. Aufl., München 2011.

Schierenbeck H., M. Lister, S. Kirmße, Ertragsorientiertes Bankmanagement - Band 2: Risiko-Controlling und integrierte Rendite-/Risikosteuerung, 9. Aufl., Wiesbaden 2009.

Wolke, T., Risikomanagement, 2. Aufl., München 2008. 


\section{Das Praxislehrbuch zum Europäischen Verbrauchsteuerrecht.}

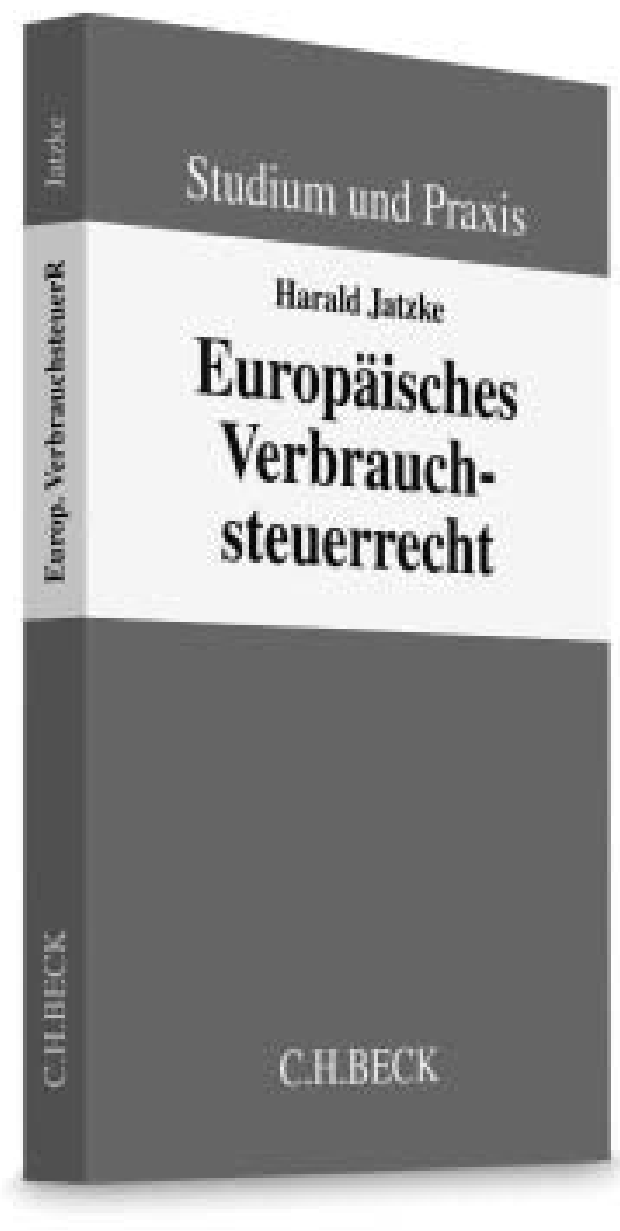

Jatzke

Europäisches Verbrauchsteuer-

recht

2016. XXII, 232 Seiten.

Kartoniert $€ 39,80$

ISBN 978-3-406-69667-1

Neu im Juni 2016

Mehr Informationen:

www.beck-shop.de/bkbrkk

\section{Schließt eine Lücke in der Fachliteratur}

Das neue Praxislehrbuch gibt einen vollständigen Überblick über das bisher kodifizierte Unionsrecht auf dem Gebiet der besonderen Verbrauchsteuern (auf Alkohol und alkoholische Getränke, auf Energieerzeugnisse und elektrischen Strom und auf Tabakwaren). Aufgrund der nahezu vollständigen Harmonisierung und der dadurch bedingten starken Prägung des nationalen Verbrauchsteuerrechts durch die Vorgaben des Unionsrechts ist das Werk eine wertvolle Arbeitshilfe, die das Verständnis der nationalen Rechtsvorschriften erleichtert und deren kritische Überprüfung ermöglicht.

\section{EuGH-Rechtsprechung vollständig einbezogen}

Wie die jüngsten Urteile belegen, hat die Rechtsprechung des EuGH erhebliche Auswirkungen auf das nationale Steuerrecht. In diesem neuen Praxislehrbuch werden daher sämtliche Urteile des EuGH in Bezug genommen, mit Leitsätzen abgedruckt und erläutert, die seit der Vollendung des Binnenmarktes am 1. Januar 1993 auf diesem Gebiet ergangen sind. Eine Einführung in das immer bedeutsamer werdende Beihilfenrecht rundet das Werk ab.

\section{Der praktische Ratgeber}

Das neue Werk richtet sich an alle, die im Studium, in der Finanzverwaltung, in der Rechtsprechung, in Herstellungsbetrieben und in der Distribution oder in der Rechtsund Steuerberatung mit Verbrauchsteuern befasst sind. 


\section{Wirtschaftsgesetze umfassend und aktuell.}

\section{Wirtschaftsgesetze}

94. Auflage. 2016. Stand April 2016. Rd. 4460 Seiten. Im Ordner $€$ 49,Ergänzungslieferungen erhalten Sie bis auf Widerruf.

ISBN 978-3-406-44957-4

Preis ohne Ergänzungslieferung $€ 79$,ISBN 978-3-406-50095-4

Neu im Juni 2016

Mehr Informationen:

www.beck-shop.de/dbd

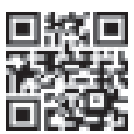

\section{Die preiswerte Sammlung}

Abgestimmt auf die besonderen Anforderungen von Wirtschaftspraxis und Wirtschaftsstudium enthält die Textsammlung mehr als 120 Gesetze, Verordnungen und Bedingungswerke aus Wirtschafts- und Arbeitsrecht, Zivilrecht mit Bankrecht, Versicherungs-, Wettbewerbs- und Insolvenzrecht, gewerblichem Rechtsschutz sowie Verfassungsrecht.

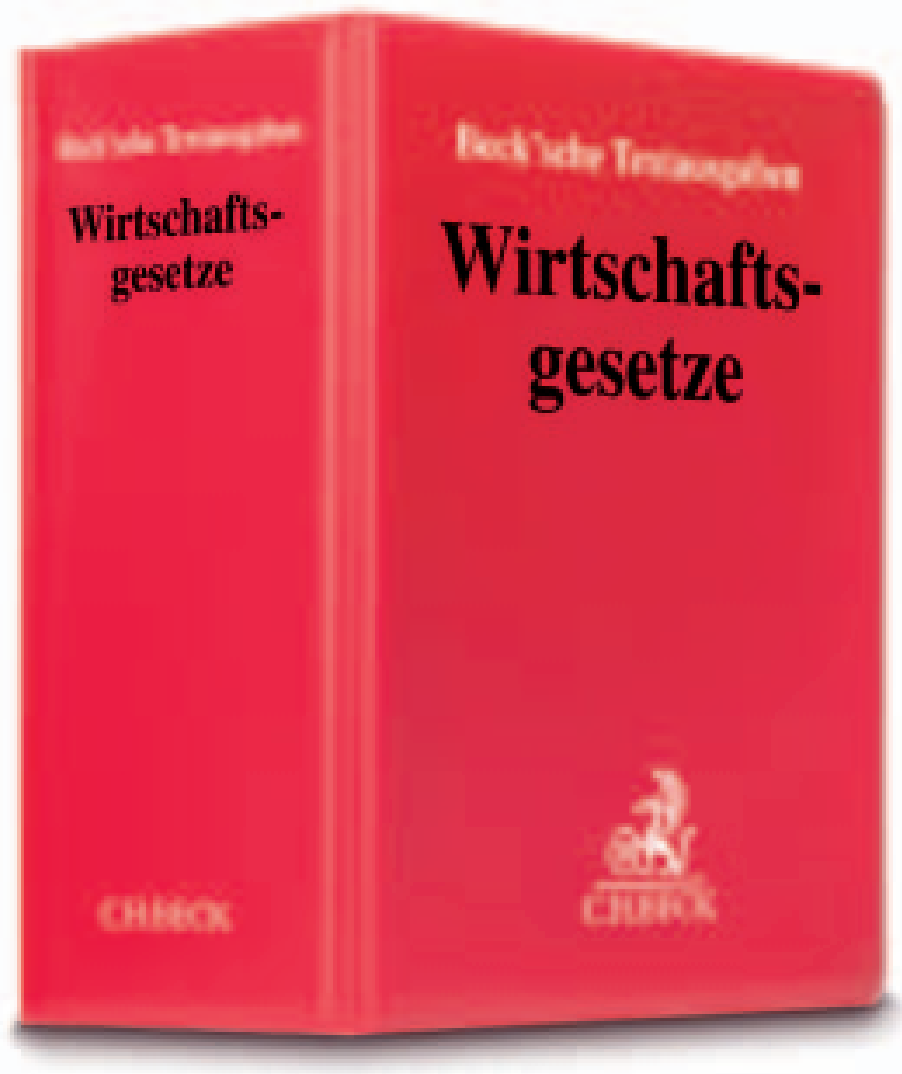

\section{Neu eingearbeitet}

mit Stand April 2016 wurden u.a.:

- VergaberechtsmodernisierungsG

- G zur Verbesserung der zivilrechtlichen Durchsetzung von verbraucherschützenden Vorschriften des Datenschutzrechts

- G zur Umsetzung der Richtlinie über alternative Streitbeilegung in Verbraucherangelegenheiten

\section{Die ideale Hilfe für:}

Juristen, Wirtschaftsfachleute, Betriebswirte, Mitarbeiter in Banken und Versicherungen, Studenten der Betriebs- und Volkswirtschaftslehre.
"Dem Wirtschaftsjuristen wie auch anderen Wirtschaftsfachleuten, Betriebswirten, Bankmitarbeitern und Studenten der Wirtschaftsdisziplinen dient die Textausgabe als täglicher Begleiter im gewohnten, roten Kleid."

RA Oliver Thum, RAe Klatte Thum, Frankfurt a.M., zur 58. Auflage 2006 


\section{Der Klassiker zur Unternehmensführung.}

\section{Dieses Lehrbuch}

stellt das gesamte Spektrum der modernen Unternehmensführung in verständlicher und praxisorientierter Form dar. Mit zahlreichen Abbildungen, Merksätzen und Anwendungsbeispielen sowie Leitfragen und Management Summaries wird es höchsten didaktischen Ansprüchen gerecht.

\section{Aus dem Inhalt}

- Grundlagen der Unternehmensführung

- Normative Unternehmensführung

- Strategische Unternehmensführung

- Planung und Kontrolle

- Organisation

- Personal

- Informationsmanagement

- Ausrichtungen der Unternehmensführung

\section{Die Neuauflage}

enthält neben vielen neuen Praxisbeispielen (u.a. Bosch, Continental, SAP, Trelleborg) auch aktuelle Themen, wie etwa Big Data und Industrie 4.0, sowie ein neues Kapitel zur innovationsorientierten Unternehmensführung.

\section{Die Autoren}

Prof. Dr. Ralf Dillerup lehrt Unternehmensführung und Controlling an der Hochschule Heilbronn. Prof. Dr. Roman Stoi lehrt Unternehmensführung und Controlling an der Dualen Hochschule Baden-Württemberg Stuttgart.

"Die Verbindung von Theorie und Praxis wird durch Beispiele geschaffen, die Denkanstöße für die tägliche Arbeit bilden."

Dr. Dietmar Voggenreiter, Mitglied des Vorstands, Marketing und Vertrieb, Audi AG

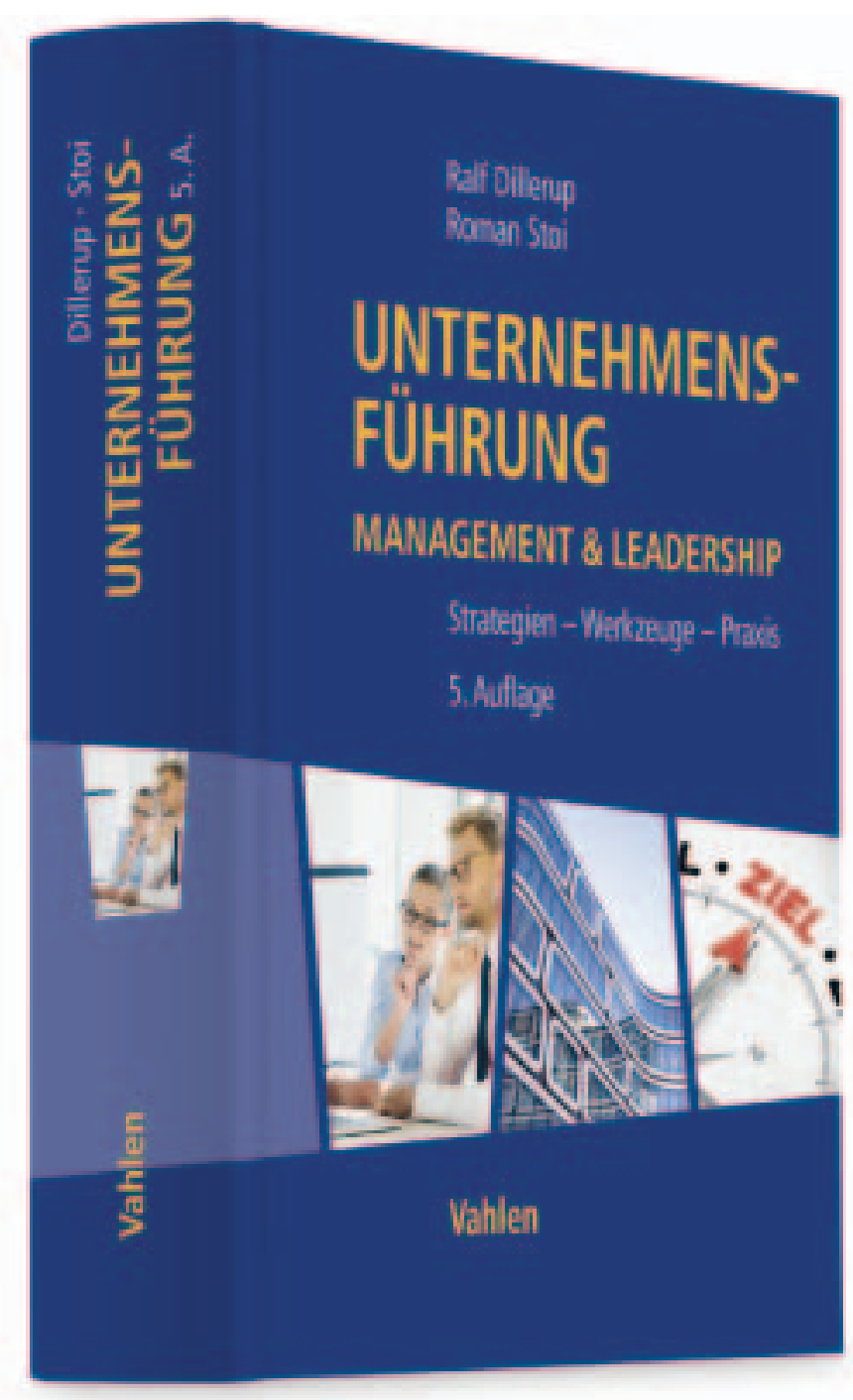

Dillerup/Stoi

Unternehmensführung

5. Auflage. 2016. Rund 1000 Seiten. Gebunden ca. $€ 49,80$ ISBN 978-3-8006-5112-2

Neu im August 2016

Portofrei geliefert: vahlen.de/15828030 